\title{
VALIDATION STUDY OF AN ANALYTICAL MODEL OF FDM ACCURACY
}

\author{
Percoco, G.; GALANTUCCI, L. M. \& LAVECChia, F.
}

Abstract: The diffusion of Additive Manufacturing technologies is leading to new needs in industrial research and development sectors. In particular, mating additive manufactured parts is not a simple task, due to the poor accuracy of several additive technologies, such as Fused Deposition Modeling (FDM). Several papers are focused on studies of how FDM accuracy is affected by process parameters. In this paper the authors report a study of the influence of working parameters on FDM dimensional accuracy, paying particular attention to variables that can affect axial symmetric mating between parts, and how these parameters can be set to improve the mating process.

Key words: fused deposition modeling, rapid prototyping, assembly
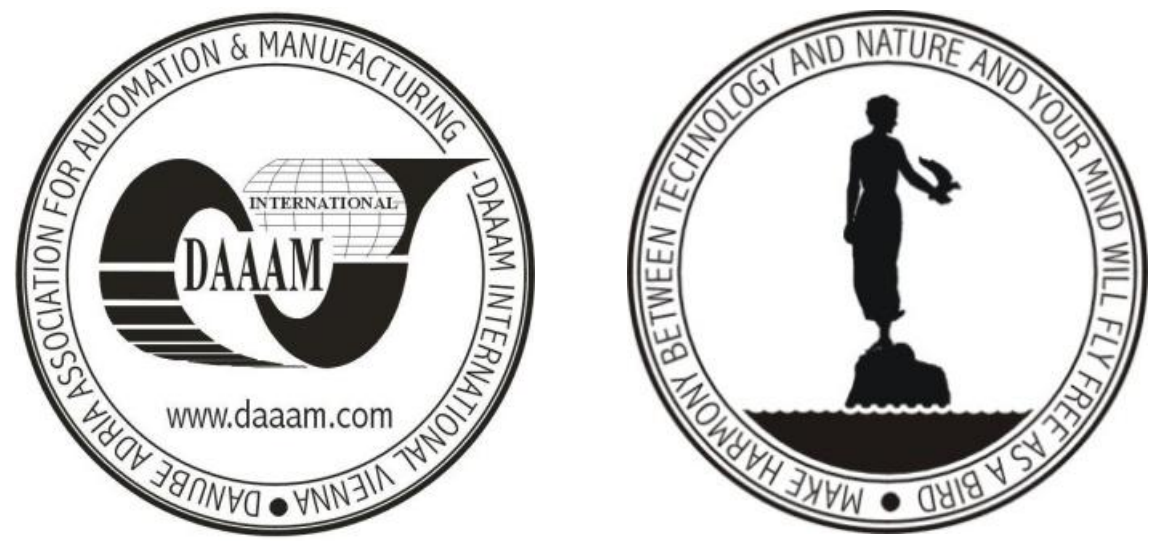

Authors' data: Ph.D. Percoco, G[ianluca]; Univ. Prof. Galantucci, L[uigi] M[aria]; Dr. Sc. Lavecchia, F[ulvio], Politecnico di Bari, Dipartimento di Ingegneria Meccanica e Gestionale, Viale Japigia 182, 70126, Bari, Italy, g.percoco@poliba.it galantucci@poliba.it f.lavecchia@poliba.it

This Publication has to be referred as: Percoco, G[ianluca], Galantucci, L[uigi] M[aria] \& Lavecchia, F[ulvio] (2011). Validation Study of an Analytical Model of FDM Accuracy, Chapter 48 in DAAAM International Scientific Book 2011, pp. 585592, B. Katalinic (Ed.), Published by DAAAM International, ISBN 978-3-90150984-1, ISSN 1726-9687, Vienna, Austria

DOI: $10.2507 /$ daaam.scibook.2011.48 


\section{Introduction}

Additive Manufacturing (AM) techniques are well known throughout the world and are often used in the manufacturing of products, as they help to significantly reduce time to market. This technological advance has been made possible thanks to research conducted by companies, universities and research centers over the years. However, the technology still needs to be further refined. In fact, several limitations have yet to be addressed and resolved, such as the poor dimensional accuracy of the products, that still limits the diffusion of AM. Among these methods, one of the most interesting is Fused Deposition Modeling ${ }^{\circledR}$ (FDM), produced by Stratasys Inc. This technology is particularly interesting because it extrudes mainly recyclable thermoplastics, thus addressing the growing concern about the environment.

Among the extrudable materials one of the most widely employed is ABS, which is ideal for conceptual prototyping through design verification, and is available in a variety of standard and custom colors.

Manufactured items ready for shipment and sale may be the final product, or sub-assembly, or components that must be assembled together. However, mating fused deposition modeled parts is not a simple task, due to the poor accuracy of this technology.

For this reason, several research studies have been focused on the goal of optimizing production parameters in order to obtain higher quality products, in terms of both surface finish and dimensional accuracy.

\section{Related research}

To quantify the capabilities of the FDM ${ }^{\circledR} 400 \mathrm{mc}^{\mathrm{TM}}$, Stratasys (Hansen, 2008), performed an in-depth analysis of its accuracy, precision and repeatability. Assessment of the dimensional accuracy was based on 108 sample parts and 2,916 measurements. The test parts were constructed on three FDM 400mc machines. The test part measured $127 \times 76$ X $14 \mathrm{~mm}$, built in ABS-M30 with standard parameters. The results of the accuracy study confirmed that the FDM 400mc produces parts to a tolerance exceeding $\pm 0.13 \mathrm{~mm}$ or $\pm 0.04 \mathrm{~mm} / \mathrm{mm}$. This specification is based on a 95\% confidence level; $96.2 \%$ of workpieces satisfied machine specifications, while of the 112 measurements that exceeded the tolerance specification, only 45 (1.54\%) were more than $0.03 \mathrm{~mm}$ beyond the allowable tolerance band.

In (Gregorian et al. 2001) the Shrinkage Compensation Factor (SCF) has been reported to influence the accuracy of FDM prototypes. As a thermoplastic material, the ABS used in FDM machines undergoes a volume reduction when heated and extruded. The SCF is an operator-controlled variable that affects the overall accuracy of the product. During production planning, shrinkage of the material is compensated by multiplying the size by the value of SCF; this is done automatically by the software once the optimal SCF has been set. After minimizing the residual error, the optimal SCF was equal to $0.7 \%$, providing low standard deviations and residual error. In conclusion, the authors suggest that assessment should be made of how changes in temperature and speed of construction, which are reflected in variations of the 
cooling time, affect the deformation. A starting point is also indicated for the study of the size of the pieces, in order to compare shrinkage on pieces of different sizes.

In (Wang et al. 2006) a model for describing Fused Deposition Modeled prototypes is proposed. In accordance with basic assumptions and simplifications, the construction of a mathematical model for deformation, containing each of the factors that influence the process, namely number of layers, length of the section, temperature of the room and shrinkage rate of the material, is presented. As regards the number of layers, the results show a strain reduction over 70 layers while the opposite trend is apparent with regard to the section length. The direct proportionality trend is also evident when assessing the degree of shrinkage of the material: the strain increases linearly with the increase of length. Finally, as regards the building temperature, the model indicates a linear reduction of the deformation increasing the chamber temperature, which becomes zero when the chamber temperature reaches the glass transition temperature of the material. However, this is only a theoretical limit because with high chamber temperatures there would be an abnormally prolonged solidification time of the material, that would severely affect the quality of the deposited layer and reduce the adhesion of the material deposited for the next layer.

In (Sood et al. 2009), the gray-Taguchi method is proposed, used to reduce several features to a single response variable, while retaining the practicality and economy of the traditional Taguchi method. The results of this study show that shrinkage along the $\mathrm{X}$ and $\mathrm{Y}$ axes is attributable to the development of internal stresses arising from the contraction of the deposited fibers. On the $\mathrm{Z}$ axis the height reduction is not systematic owing to the discretization due to the slice height. During cooling residual stresses proportional to the thermal shrinkage coefficient and nonuniform temperature gradient over the piece are experienced. The results show that among the critical factors, the most important is the building direction of the piece, followed by the layer thickness and angle of the raster, and then, to a lesser extent, the air gap and raster width.

In (Zang \& Chou, 2008), a finite element model that simulates the FDM process is proposed. This model performs mechanical and thermal analysis, allowing evaluation of the phenomenon of accumulation of residual stresses within the prototypes due to repeated heating and cooling cycles during the construction process. This model is applied to investigate the effects of key parameters such as the deposition rate, layer thickness and road width, repeated on three levels. The first parameter is responsible for the thermal cycles, resulting in different thermal gradients in the piece causing residual stresses. The thickness of the layer road width affects the construction speed of the piece: if the layer is thick there will be fewer thermal cycles. The response variable considered is the equivalent stress accumulated during the deposition, which is closely related to the deformation of the workpiece. Following statistical analysis of the results obtained by measuring the parts produced by a CMM machine, the authors concluded that the deposition rate has the strongest influence, followed by the layer thickness.

Recent improvements in FDM, such as the possibility of using sterilizable materials and the improvements in accuracy, have opened out new applications in 
new fields, such as reconstructive surgery. However, these new fields require good tolerances, too. For example, in (El-Katatny et al. 2010) the aim of the paper is to study the error generated in the construction of complex anatomical reproductions, using CT scans. An evaluation of dimensional errors is conducted, focusing mainly on two body parts, skulls and mandibles of different sizes (child, woman, man). Comparison was made of 11 measurements on the virtual model of a skull and 9 on the mandible of a patient with those related to the corresponding replicas produced with FDM. Although the technology demonstrated its ability to produce prostheses adapted to the specific proportions of a patient quickly and relatively cheaply, the dimensional accuracy is a key factor. The results showed a smaller product than the nominal sizes, with a mean absolute deviation of $0.24 \%$ and a standard deviation of $0.16 \%$ for the skulls, and $0.22 \%$ and $0.11 \%$ for the jaws.

There are ample reports in literature about the accuracy of FDM prototypes but there are no studies addressing the problem of mating of the components. In this work the authors focus on the accuracy of FDM prototypes, making an in-depth study of aspects that are important in assembly tasks, such as the presence of clearance or interference between workpieces to be mated together.

\section{Proposed Approach}

The mathematical model presented in literature (Wang et al. 2006) is applied and tested in preliminary experiments. Subsequently, an in-depth experimental study is proposed, to analyze and predict the true dimension of shaft-hole prototypes needing to be assembled together. In the hypothesis that the inner stresses of the fibers are mainly produced in the process of the fibers' temperature changing from the glass-transition temperature $\mathrm{Tg}$ to the chamber temperature $\mathrm{Te}$, it is possible to compute the curvature radius $\mathrm{R}$ as (Wang et al. 2006):

$$
R=\frac{1}{\frac{6 \alpha}{h}\left(T_{g}-T_{e}\right)\left(1-\frac{s}{h}\right) \frac{s}{h}}
$$

where $s$ is the slice height, $h$ is the slice quota whose distortion must be computed and $\alpha$ is the shrinkage coefficient. Once the curvature radius has been computed, the new length $L^{\prime}$ ' of the slice, if $\delta \cong 0$, can be computed as (Figure 1):

$$
\mathrm{L}^{\prime}=2 \sqrt{\left(\mathrm{R}^{2}-(\mathrm{R}-\delta)^{2}\right.}
$$

with:

$$
\delta=\mathrm{R}\left(1-\cos \left(\frac{\mathrm{h}}{2 \mathrm{R}}\right)\right)
$$

The model has been tested on the following specimens: (i) one cylinder, base diameter equal to $50 \mathrm{~mm}$, height $15 \mathrm{~mm}$; (ii) one cylinder, base diameter equal to 80 
$\mathrm{mm}$, height $15 \mathrm{~mm}$, central through hole of diameter $50 \mathrm{~mm}$; (iii) one plate 140x140x15 mm $\mathrm{mm}^{3}$, with a central through hole, diameter equal to $50 \mathrm{~mm}$ (Figure 2).

Each specimen has been manufactured in twelve copies, six have undergone a support removal in water and six a manual support removal; each group of six has been subdivided into three manufactures at full density (solid) and three at low density (sparse).

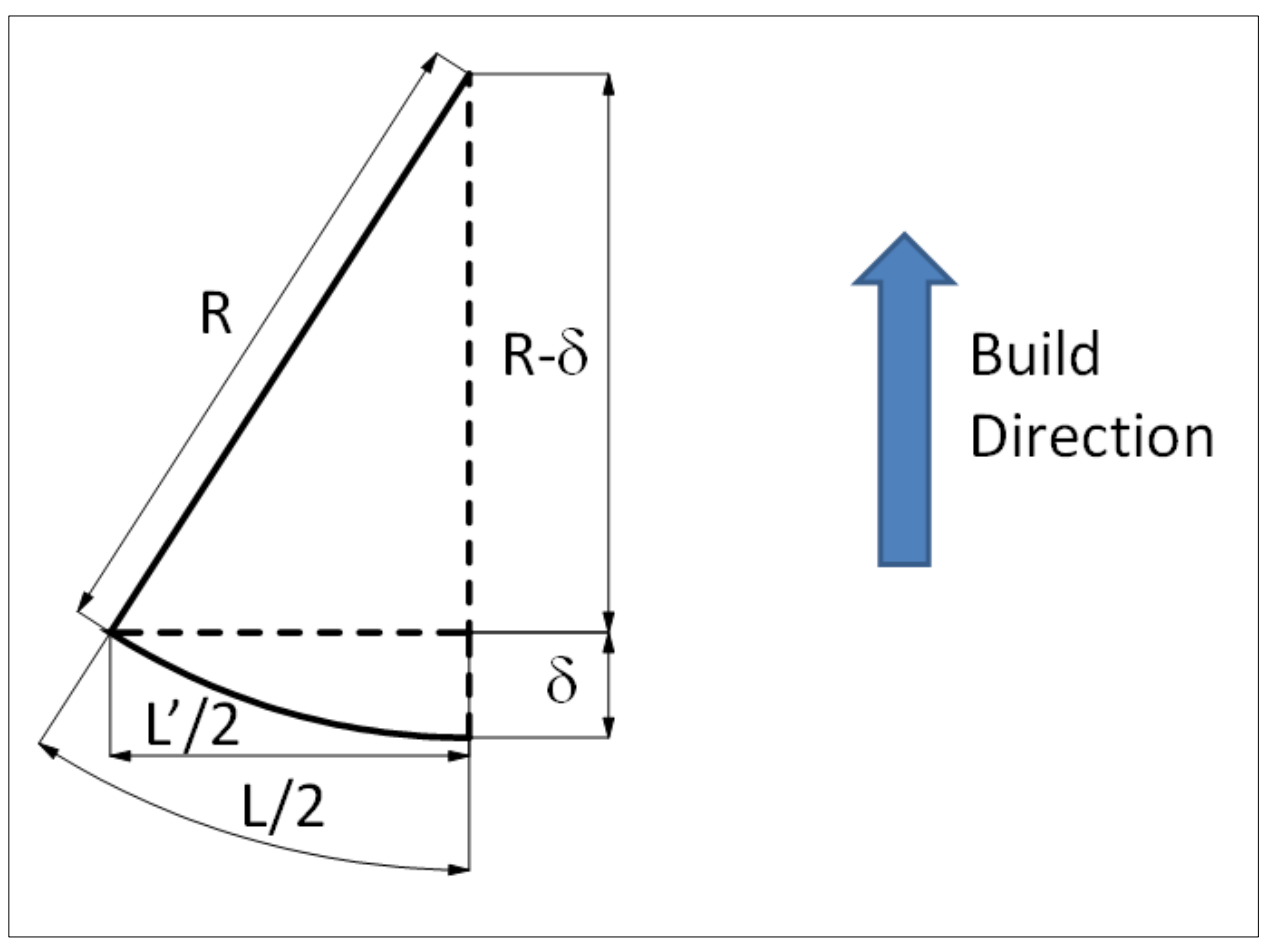

Fig. 1. Geometric considerations about slice distortion

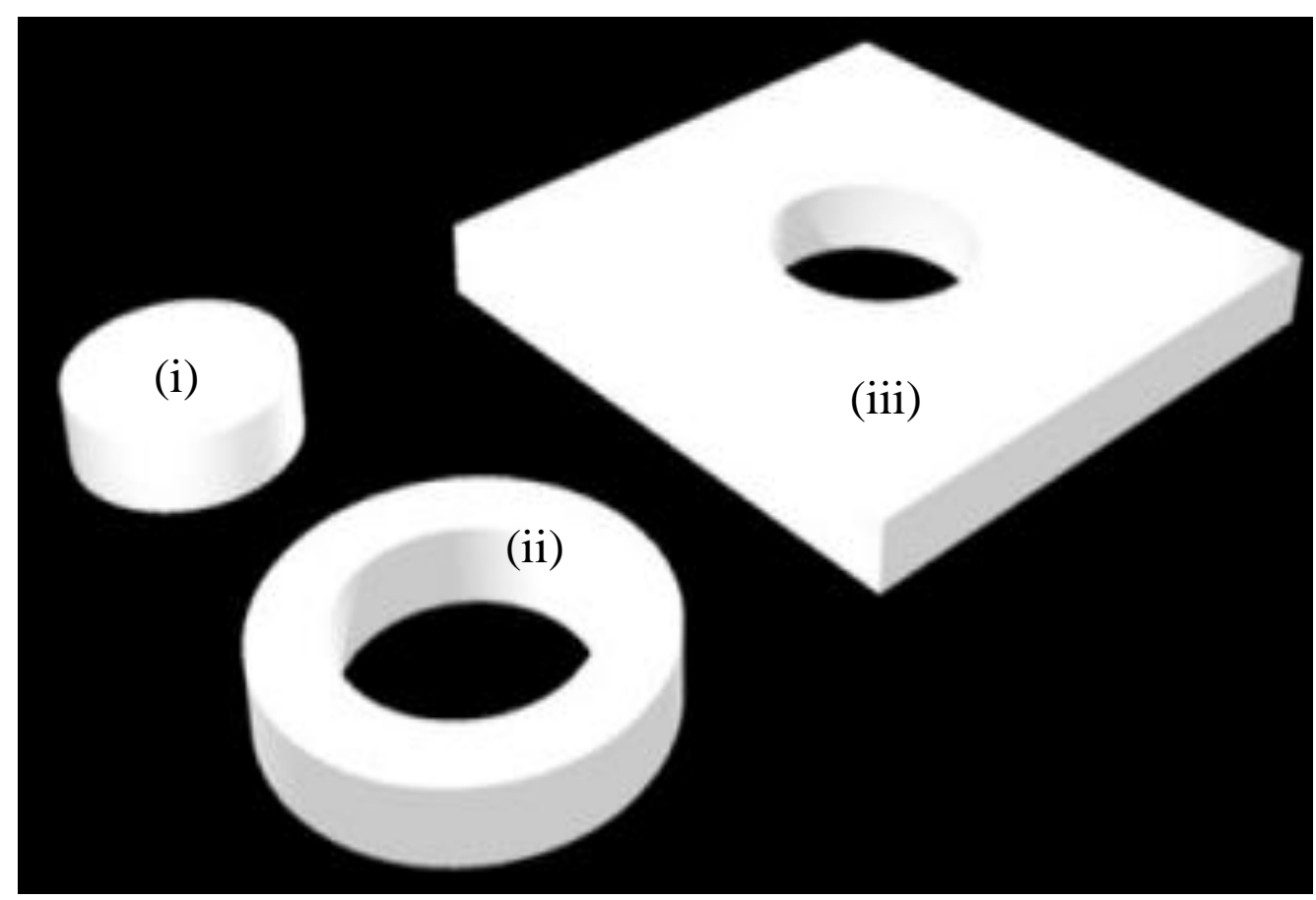

Fig. 2. Specimens considered for the experimentation 
For the sake of brevity only the results related to the dimension of the internal holes of specimens (ii) and (iii) are shown in Table 1. Generally speaking it can be said that full density specimens shrink more than low density specimens, watertreated specimens have very similar shrinkage to break-away supports and that the mathematical model shows a better performance on full density workpieces. Moreover, as regards specimens (ii) and (iii) the external measured dimension shows no significant variation from the nominal value.

Because the mathematical model limits are related especially to sparse workpieces, and these kind of workpieces are the most commonly used for fabrication, it was deemed necessary to devise an experimental approach evaluating manufacturing tolerances of shaft-hole mating workpieces after an exact definition of the input variables, and determination of the shape and size of the pieces to be produced. The experimental plan studied specimens (i) and (ii), including their density. Moreover, in view of reports in literature, the chamber temperature and several specimen dimensions were also considered, to verify the possibility of managing this technology for the production of prototypes to be assembled together.

\begin{tabular}{|lllll|}
\hline Water removal of supports & Solid & Sparse & Model & Nominal \\
\hline Shaft diameter (i) & 49.88 & 50.01 & 49.85 & 50 \\
Hole diameter (ii) & 49.86 & 49.98 & 50.61 & 50 \\
Hole diameter in plate (iii) & 50.01 & 50.05 & 50.86 & 50 \\
\hline Break away support & & & & \\
Shaft diameter (i) & 49.91 & 50.02 & 49.85 & 50 \\
Hole diameter (ii) & 50.01 & 49.98 & 50.61 & 50 \\
Hole diameter in plate (iii) & 49.89 & 50.06 & 50.86 & 50 \\
\hline
\end{tabular}

Tab. 1. Measured diameters vs calculated diameters

Firstly (a), the temperature of the processing chamber, subdivided into three levels, namely $65{ }^{\circ} \mathrm{C}, 70{ }^{\circ} \mathrm{C}$ and $75^{\circ} \mathrm{C}$; the main reason for considering this variable lies in the influence it has on the cooling rate of the material.

The P400 ABS for example, takes about 0.55 seconds to cool from the melting temperature of $270{ }^{\circ} \mathrm{C}$ to the glass transition temperature of $94{ }^{\circ} \mathrm{C}$ (considering a nozzle diameter of $0.254 \mathrm{~mm}$ ), and then takes 1.2 seconds from the glass transition temperature to the working environment, equal to $70{ }^{\circ} \mathrm{C}$. Then (b), the air gap, namely the space between two adjacent roads on the same layer. This factor was studied on two levels: 0 and $0.008 \mathrm{~mm}$, to verify whether the presence of air between the strands of material can affect cooling of the ABS. Moreover, acting on the air gap can make the piece more or less dense, and increasing the quantity of material can result in a higher absorption of moisture from the environment. Finally (c), the size of the workpieces, studied on three levels, for specimens (i) at a diameter equal to $50,37.5$ and $25 \mathrm{~mm}$ and for specimens (ii) at external/internal diameters equal to $80 / 50,60 / 37.5,40 / 25 \mathrm{~mm}$. 
The primary interest in this variable is the relation between shrinkage and dimension, that is not manageable with shrinkage. theory due to the high anisotropy of workpieces.

\section{Results}

The response variables chosen to evaluate the results were shaft deviation, i.e. the difference between the nominal value and average measured value of the shaft diameters for specimens (i), and hole deviation, that is the difference between the nominal value and average measured value of the internal diameters of specimens (ii). A mixed level full factorial design, with three replications, was chosen. Such a plan is best suited to the need for detailed information typical of an exploratory experiment. With this type of plan, two factors are studied on three levels and the Air Gap only on two levels.

Eighteen different combinations were generated, repeated three times, producing 108 pairs of specimens. Each specimen was measured on three different $\mathrm{z}$ levels and each measurement was repeated three times. As a consequence each specimen underwent nine diameter measurements. The mean of the nine measurements was the diameter taken as the response variable. The experimental plan was analyzed using Minitab statistical software. Firstly, the standardized effects of the input variables were studied on both the response variables. In Figure 3, left side, the effects of the input variables on the differences between the nominal and measured values for specimens (i) are shown. The higher effects are due to second and third order interactions, but with a confidence level of less than $99 \%$.
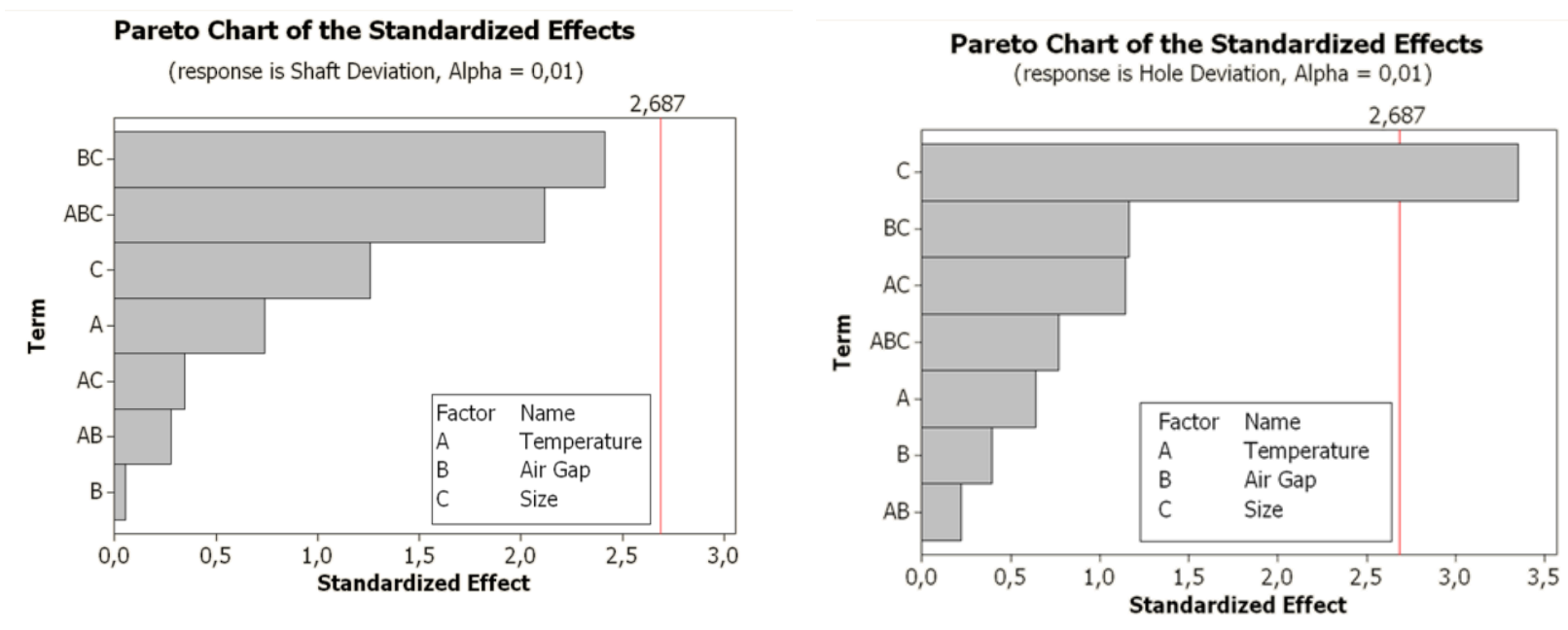

Fig. 3. Standardized Effects on Shaft Deviation (left) and Hole Deviation (right)

In Figure 3, right side, the Pareto Chart of the standardized effects on the measured internal diameters of specimens (ii) is shown. The strong effect of the diameter size on the hole deviation is evident, with a confidence level of more than 99\%. This phenomenon is probably due to the poor behaviour of the Stratasys shrink compensation factor on hollow workpieces. As a consequence, workpieces with 
cavities need to be accurately studied to improve mating, since it is only the diameter size that affects measured differences in shafts and holes.

\section{Conclusion}

In this paper the fabrication tolerances of FDM prototypes have been studied using an experimental approach, oriented to shaft-hole mating. The experimentation has been conducted on two levels, the first one to verify a theoretical approach found in literature that showed to be adequate essentially for work-pieces fabricated with the solid strategy and without cavities. As a consequence, the second experimental level was conducted to identify factors influencing the accuracy of production of workpieces with cavities and sparse deposition strategies. Hollow parts resulted to be influenced essentially by the size of the hole, whereas the shafts were not affected by the size, probably due to a correct application of the shrinkage correction factor. A shrinkage model from literature has been tested giving correct results with solid parts but poor results for hollow and sparse parts.

\section{References}

El-Katatny, I., Masood, S.-H., Morsi, Y.-S., 2010, Error Analysis of FDM Fabricated Medical Replicas, Rapid Prototyping Journal, Vol. 16, No 1, pp. 36 - 43, ISSN: $1355-2546$

Gregorian, A., Elliot, B., Navarro, R., Ochoa, F., Singh, H., (2001) Accuracy Improvement in Rapid Prototyping Machine (FDM-1650), Available from: http://utwired.engr.utexas.edu/lff/symposium/preceedingsArchive/Manuscripts/ 2001/2001-10-Gregorian,Noorani.pdf, Accessed on: 2011-07-01

Hansen, J., 2008, FDM 400mc Accuracy Assesment, Available from: http://www.protech.se/ele-

ments/common/files/docs/FDM400mc_Accuracy.pdf, Accessed on: 2011-0701

Sood, A.-K., Ohdar, R.-K., Mahapatra, S.-S., 2009, Improving Dimensional Accuracy of Fused Deposition Modelling Part Using Grey Taguchi Method, Materials \& Design, Vol. 30, No 10, pp. 4243-4252, ISSN: 0261-3069

Wang, T.-M., XI, J.-T., Jin, Y., 2006, A Model Research for Prototype Warp Deformation, The International Journal of Advanced Manufacturing Technology, Vol. 33, No 11-12: pp. 1087-1096, ISSN: 1433-3015

Zang, Y., Chou, Y.-K., 2008, A Parametric Study of Part Distortions in Fused Deposition Modelling Using Three-Dimensional Finite Element Analysis, Proceedings of the Institution of Mechanical Engineers, Part B: Journal of Engineering Manufacture, Vol. 222, No. 8, pp. 959-968, ISSN: 2041-2975 